\title{
Altered oxidative stress levels in Indian Parkinson's disease patients with PARK2 mutations
}

\author{
Monika Vinish ${ }^{\star}$, Akshay Anand ${ }^{\star}$ and Sudesh Prabhakar ${ }^{\bigotimes}$ \\ Department of Neurology, PostGraduate Institute of Medical Education and Research (PGIMER), Sector-12, Chandigarh, India
}

\begin{abstract}
The aim of this pilot study was to determine the baseline state of oxidative stress indices in patients with Parkinson's disease (PD). Peripheral blood samples of 15 PD subjects were analyzed and compared with ten age matched healthy controls. Patients with PARK2 mutations were also compared with PD patients without mutations. There was significant increase in malondialdehyde content and superoxide-dismutase (SOD) activity in peripheral blood parameters in PD patients $(P<0.05)$ in comparison to controls. These findings suggest an important role of oxidative stress in Parkinson's disease evolution and progress. No changes were observed in glutathione peroxidase and nitric oxide levels. We found significant correlation between SOD activity and lipid peroxidation when the biochemical data was further analyzed. In addition, significant increase in the levels of SOD among the PD patients with PARK2 mutations was observed, which can be ascribed to chronic oxidative stress induced by PARK2 mutations.
\end{abstract}

Key words: oxidative stress, mutations, Parkinson's disease, PARK2

Received: 19 November, 2009; revised: 24 January, 2011; accepted: 08 May, 2011; available on-line: 17 May, 2011

\section{INTRODUCTION}

Parkinson's disease (PD) is characterized by a loss of dopaminergic neurons in the substantia nigra, leading to the major clinical and pharmacological abnormalities that characterize the disease. Although the pathogenesis of PD remains ambiguous, oxidative stress (OS) to dopaminergic neurons in the substantia nigra pars compacta $(\mathrm{SNpc}$ ) has been reported to be one of the leading causes of neurodegeneration in PD (Bahmann et al., 2004). The human body has evolved several defense mechanisms to counteract OS such as vitamin E, vitamin $\mathrm{C}$, vitamin A, glutathione and various antioxidant enzymes, but the brain appears to be more susceptible to these assaults than other organs because of its low antioxidant capacity. Being highly metabolic, brain tissue generates more oxyradicals. Alteration in the oxidative stress has been proposed to cause the loss of dopaminergic neurons in PD patients (Hung \& Lee, 1998). Although the changes in lipid peroxidation and antioxidant defenses are documented in the SNpc of PD patients but there is difficulty in obtaining a brain biopsy, until after the death of the afflicted individual. It is therefore crucial to develop suitable peripheral markers, which can help in the diagnosis of PD during life.

The etiology of Parkinson's disease is unknown although both genetic susceptibility and environmental factors appear to play an important role in its development. Parkin is a Parkinson disease-related E3 ubiquitin ligase; parkin-deficient animals exhibit mitochondrial degeneration and increased oxidative stress vulnerability, and both mice and flies lacking DJ-1 are hypersensitive to environmental toxins associated with PD (Palacino et al., 2004; Shen \& Cookson, 2004). Currently, accumulating evidence indicates that parkin may play a role in maintaining mitochondrial function and preventing oxidative stress (Hyun et al., 2005). We therefore examined if $P A R K 2$ deletions alter the antioxidant profile of Indian PD patients.

\section{MATERIAL AND METHODS}

Patients. The study group included 15 sporadic or non-consanguineous PD patients visiting the Neurology Clinic at the PostGraduate Institute of Medical Education and Research (Chandigarh, India) and ten healthy controls. The diagnosis of Parkinson's disease was made on the basis of the UK Parkinson's Disease Society Brain Bank Research criteria, London (Hughes et al., 1992). Clinical diagnosis was established with the presence of at least two of the cardinal symptoms, i.e., tremors, muscular rigidity, bradykinesia and postural instability while patients with vertical gauge impairment, marked autonomic disturbances, atypical Parkinsonism and those on antipsychotic drugs were excluded from the study (Lang \& Lozano, 1998). Written informed consent was obtained from all patients and controls as per the Institute Ethics Committee guidelines. Genetically unrelated controls were also examined for the absence of extra-pyramidal signs, which included spouse of the patient and other age, sex and ethnicity matched healthy individuals. The mean age of onset for patients recruited for the study was $46.5 \pm 2.1$ years while that for healthy volunteers was $43.4 \pm 2.2$. About $10 \mathrm{~mL}$ of venous blood was drawn for genetic analysis from these patients and controls. All the biochemical assays were performed in duplicates.

Superoxide dismutase (SOD) assay. Determination of $\mathrm{Cu}, \mathrm{Zn}$-SOD activity was performed using a commercial kit (Ransod; Randox, CrumLin, UK) based on the method developed by McCord and Fridovich (1988). Coupling of $\mathrm{O}_{2} \cdot$ generators (xanthine and xanthine oxi-

\footnotetext{
e-mail: pgineurology@gmail.com

These authors contributed equally to this work

Abbreviations: $\mathrm{Gp}_{x^{\prime}}$, glutathione peroxidase; INT, (2-(4-iodophenyl)3-(4-nitrophenol)-5-phenyltetrazolium chloride LRRK2, leucine rich repeat kinase; MDA, malondialdehyde; PD, Parkinson's disease; PARK2, Parkin gene; SNc, substantia nigra; SOD, superoxide dismutase; PINK-1, PTEN-induced putative kinase 1
} 
dase) with an $\mathrm{O}_{2} \cdot$ detector INT (2-(4-iodophenyl)-3-(4nitrophenol)-5-phenyltetrazolium chloride) leads to formation of red formazan dye. One unit of SOD activity was defined as the amount of protein that inhibits the rate of INT reduction by $50 \%$. Enzyme activity was measured in SOD units $/ \mathrm{mL}$ of whole blood.

Glutathione peroxidase $\left(\mathrm{Gp}_{\mathrm{x}}\right)$ assay. Measurement of glutathione peroxidase $\left(\mathrm{G}_{\mathrm{x}}\right)$ activity was performed using Ransel reagents (Randox Laboratories, UK) and is based on the method of Paglia and Valentine (1967). $G p_{x}$ catalyzes the oxidation of glutathione (GSH) by cumene hydroperoxide. In the presence of glutathione reductase (GR) and NADPH the oxidized glutathione (GSSG) is immediately converted into the reduced form with a simultaneous oxidation of NADPH to $\mathrm{NADP}^{+}$. For the $G p_{x}$ assay, hemolysates $(50 \mu \mathrm{L})$ were diluted with $1.0 \mathrm{~mL}$ of Ransel diluting agent and incubated for 5 minutes, followed by the addition of $1.0 \mathrm{~mL}$ of the Drabkin reagent. The decrease in absorbance at $340 \mathrm{~nm}$ was measured.

Nitric oxide estimation. Nitric oxide estimation was done by the method of Titheradge (1998). About $100 \mu \mathrm{L}$ of the sample (plasma) was added to $400 \mu \mathrm{L}$ of distilled water, $500 \mu \mathrm{L}$ of freshly prepared solution $C$ (Griess reagent) was then added to the vials containing sample. The reaction mixture was incubated at room temperature for 10 minutes and absorbance was read at $546 \mathrm{~nm}$.

Lipid peroxidation. Brain tissues are rich in phospholipids and vulnerable to attack by oxygen-derived free radicals to initiate lipid peroxidation. Lipid peroxidation was evaluated as the concentration of malondialdehyde (MDA), a lipid peroxidation end product that reacts with TBA (thiobarbituric acid) to form a conjugate. MDA levels thus provide valuable information for evaluation of oxygen radical-induced oxidative stress. In our study, MDA was assayed by the method of Buege and Aust (1978). About $1.0 \mathrm{~mL}$ of plasma was mixed with $2 \mathrm{~mL}$ of mildly heated reagent $(15 \%(\mathrm{w} / \mathrm{v})$ trichloroacetic acid, $0.375 \%(\mathrm{w} / \mathrm{v}) \mathrm{TBA}$, $0.25 \mathrm{M} \mathrm{HCl}$ ). The solution containing plasma and reagent was heated in boiling water bath for $15 \mathrm{~min}$ and then cooled. The flocculation precipitate was removed by centrifugation at 1000 r.p.m. for $10 \mathrm{~min}$. The supernatant was collected and the absorbance was read at $535 \mathrm{~nm}$ against appropriate blank. The amount of MDA was calculated using molar absorption coefficient of MDA $\left(1.56 \times 10^{5} \mathrm{M}^{-1} \cdot \mathrm{cm}^{-1}\right)$. The results were expressed as mmol of $\mathrm{MDA} / \mathrm{L}$.

PARK2 analysis. Genomic DNA was extracted from the sporadic PD patients and controls. All exons of PARK2 were amplified by PCR using parkin specific primers (Kitada et al., 1998). PCR products were visualized on $2 \%$ agarose gel for absence or presence of amplified product. Gene dosage analysis was not carried out because lack of real-time PCR device in our institute. Therefore this study was restricted to qualitative analysis alone.

In order to determine the mutations in PARK2, PCR amplified exons were subjected to SSCP and band pattern was obtained by silver staining. Sequencing was performed for all those PCR products of patients and controls that showed mobility shift by SSCP analysis.

Statistical analysis. The mean values of various antioxidants were compared using Student's $t$-test. Correlation between different variables was tested using nonparametric Spearman's coefficient and statistical significance was considered at $P<0.05$. (SPSS 17.0, Chicago, IL, USA).

\section{RESULTS}

Central Nervous System related studies have been confronted with the complexity of direct investigation because of difficulty in obtaining biopsies. It is therefore crucial to investigate peripheral markers, which can help in the early diagnosis of Parkinson's disease (PD). Our recent study has revealed upregulated levels of SOD and lipid peroxidation in PD patients (Sharma et al., 2008). Molecular studies in familial forms of the disease have identified genes: $\alpha$-synuclein, parkin, DJ-1 (PARK7), PINK-1 (PTEN-Induced putative kinase 1) and LRRK2 (leucine rich repeat kinase) encoding key proteins involved in PD pathogenesis, and support a major role for mitochondrial dysfunction and oxidative stress (Thomas $\&$ Beal, 2007). Here we studied the antioxidant profile (SOD, G $\mathrm{p}_{\mathrm{x}}$, nitric oxide and lipid peroxidation (MDA) in blood of PD patients and controls using spectrophotometric analysis.

\section{Superoxide dismutase (SOD)}

SOD is a $\mathrm{Cu}, \mathrm{Zn}$ containing enzyme responsible for catalytic dismutation of highly reactive and potentially toxic superoxide radicals $\left(\mathrm{O}_{2}^{-\cdot}\right)$ to $\mathrm{H}_{2} \mathrm{O}_{2}$ (McCord and Fridovich, 1988). $\mathrm{O}_{2}^{-\cdot}$ is constantly generated in the body tissues and failure in its removal can initiate a damaging effect on polyunsaturated fatty acids and structural proteins of plasma membranes. We estimated SOD levels among PD patients and controls using spectrophotometric analysis. The results showed significantly increased SOD levels $(P=0.026)$ in PD patients as compared to controls $(n=10)$ (Fig. 1).

\section{Glutathione peroxidase $\left(G p_{x}\right)$}

$G p_{x}$ has been previously reported as an important hydroperoxide-degrading enzyme and the importance of glutathione reductase (GR) lies in its ability to keep glutathione in its reduced (biologically active) form. Numerous chemical processes in aerobic cells lead to the production of peroxides by activated forms of oxygen. These peroxides by their decomposition to free radicals and other reactive chemical species cause oxidative damage in biological tissues. The simplest hydroperoxides such as $\mathrm{H}_{2} \mathrm{O}_{2}$ and lipid peroxides can be detoxified by the selenium-dependent $\mathrm{Gp}_{\mathrm{x}}$. The activity was estimated by following the oxidation of NADPH, required for the reduction of GSSG to GSH. We only found an insignificant decrease in the $G p_{x}$ levels $(699 \pm 59.4)$ in patients as compared to controls (730 \pm 79.0$)$ (Fig. 2).

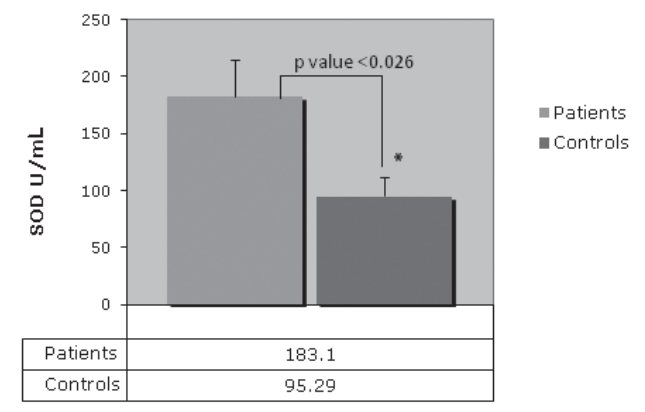

Figure 1. SOD levels in PD patients and controls. 


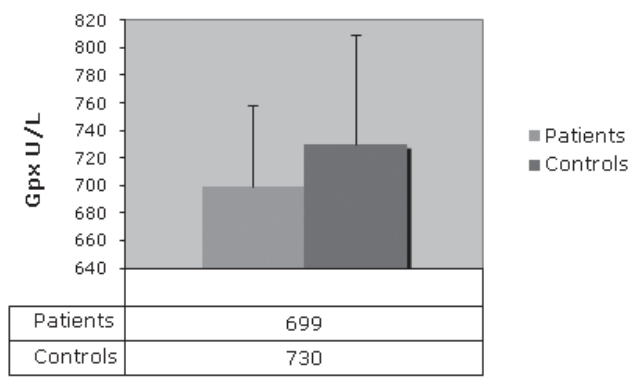

Figure 2. Glutathione peroxidase $\left(G p_{x}\right)$ levels in PD patients and controls

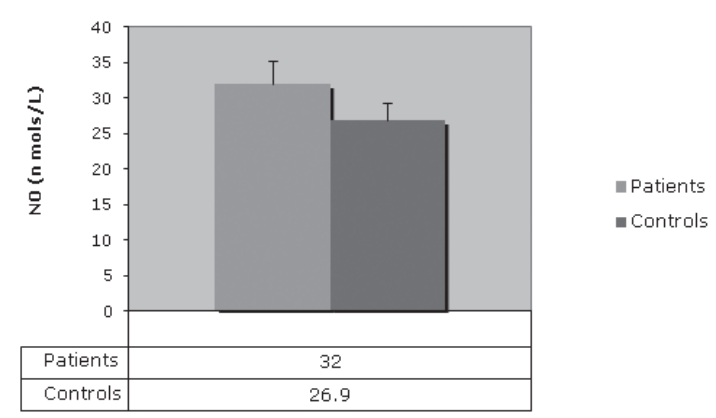

Figure 3. Nitric oxide levels in PD patients and controls

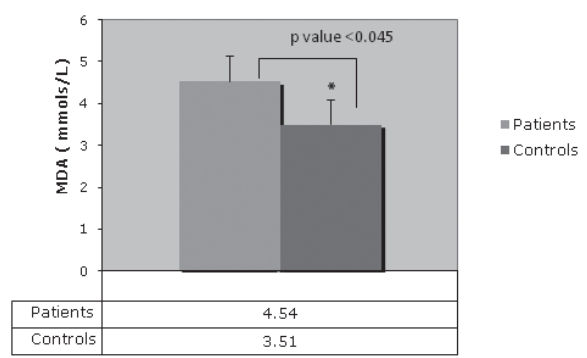

Figure 4. Lipid peroxidation (MDA) levels in PD patients and controls

\section{Nitric oxide estimation}

Nitric oxide is a unique biological messenger molecule which plays diverse physiologic roles. NO mediates blood vessel relaxation, immune activity of macrophages and neurotransmission of central and peripheral neurons. Our results revealed a slight increase in NO levels in PD patients $(32.0 \pm 3.3)$ vs controls $(26.9 \pm 2.4)$ but this was statistically insignificant (Fig. 3).

\section{Lipid peroxidation}

Lipid peroxidation reflects oxidative deterioration of polyunsaturated fatty acids, important constituents of biological membranes and is measured in terms of nmols of MDA formed $/ \mathrm{mg}$ protein. Higher levels of MDA, a marker of oxidative stress, have been reported in the SNpc of PD patients (Dexter et al., 1989). Increased lipid peroxidation is well reported in neurodegenerative diseases (Dei et al., 2002). Similarly, we found a significant increase in the plasma MDA levels of PD patients as compared to controls (Fig. 4). Thus these increased lipid peroxidation products suggest that ROS have an impor-

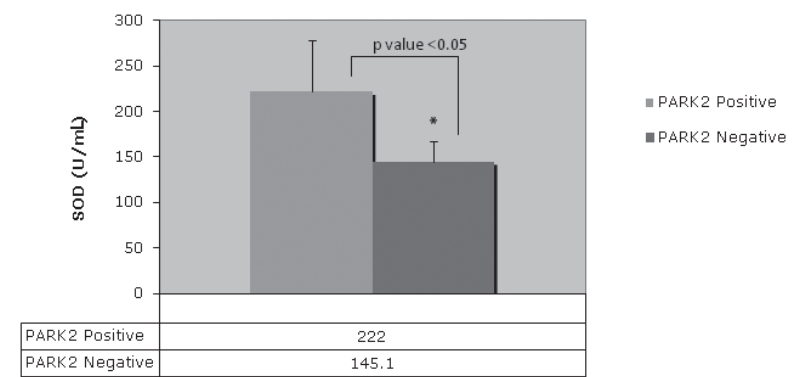

Figure 5. SOD levels in PD patients with and without PARK2 mutations

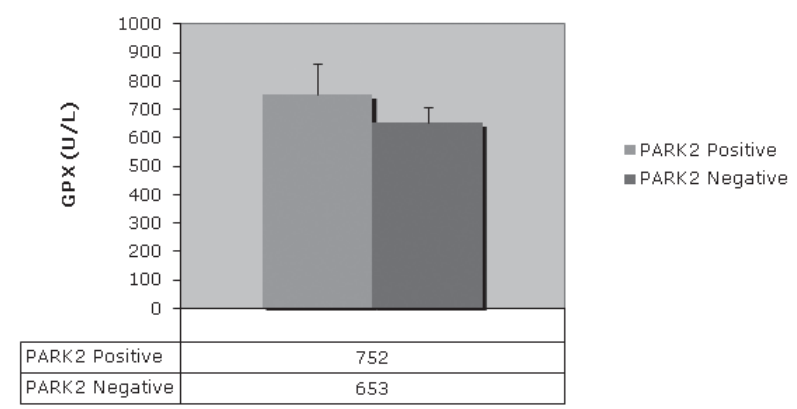

Figure 6. Glutathione peroxidase $\left(G p_{x}\right)$ levels in PD patients with and without PARK2 mutations

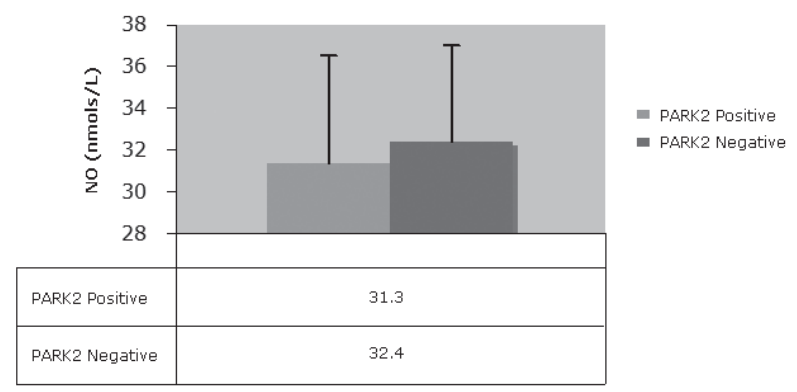

Figure 7. Nitric oxide levels in PD patients with and without PARK2 mutations

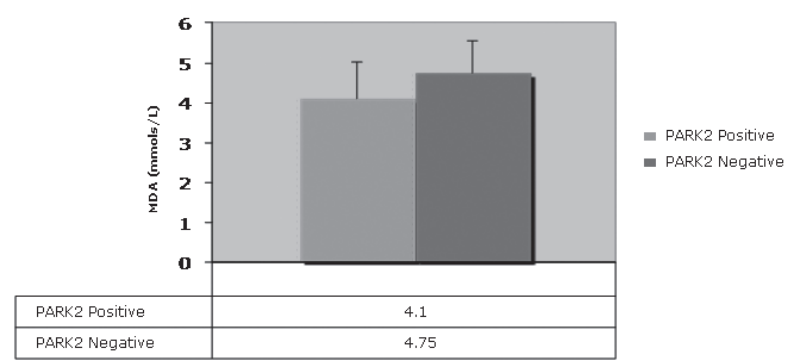

Figure 8. Lipid Peroxidation (MDA) levels in PD patients with and without PARK2 mutations.

tant role to play in the pathogenesis of neurodegenerative diseases.

\section{PARK2 analysis}

All the 12 exons of PARK2 were amplified in PD patients and healthy controls. Absence of a band was confirmed by repeating PCR and revalidated by GAPDH 
Table 1. Correlation among the various biochemical variables

\begin{tabular}{lrrrrrr}
\hline Variables & \multicolumn{1}{l}{ Sex } & \multicolumn{1}{l}{ Age } & \multicolumn{1}{l}{ SOD } & \multicolumn{1}{l}{$\mathrm{Gp}_{\mathrm{x}}$} & \multicolumn{1}{l}{$\mathrm{Lpx}$} & \multicolumn{1}{l}{$\mathrm{NO}$} \\
\hline Sex & 1.00 & 0.175 & 0.456 & 0.171 & -0.116 & 0.463 \\
Age & 0.175 & 1.0 & -0.378 & -0.113 & -0.507 & 0.031 \\
SOD & -0.456 & -0.378 & 1.0 & -0.093 & $0.797^{* *}$ & -0.187 \\
Gp & 0.171 & -0.113 & -0.093 & 1.0 & -0.225 & 0.341 \\
Lpx & -0.116 & -0.507 & $0.797^{* *}$ & -0.225 & 1.0 & -0.279 \\
NO & 0.463 & 0.031 & -0.187 & 0.341 & -0.279 & 1.0 \\
\hline
\end{tabular}

${ }^{* *}$ Correlation is significant at $P<0.01$ level (2-tailed)

(positive control) amplification of the same template. $P A R K 2$ analysis in these patients revealed exonic deletions in exons 1, 2, 3 and 12 by PCR and SSCP analysis. The exons were amplified thrice under same set of PCR conditions. In addition, when the biochemical values of these PD patients carrying $P A R K 2$ mutations and those without mutations were compared (Figs. 5-8), a significant increase in the levels of SOD among the PD patients with PARK2 mutations was observed, which can be attributed to chronic oxidative stress induced by PARK2 mutations.

\section{Correlation between biochemical parameters}

Spearman correlation analysis was performed to study the association between the biochemical parameters. A significant positive correlation between superoxide dismutase and MDA levels was found $(P=0.001)$ (Table 1$)$.

\section{DISCUSSION}

Oxidative stress represents one of the risk factors that can promote neurodegeneration in PD. Recent evidence suggests that several known mutations cause familial Alzheimer disease (AD) (amyloid $\beta$ protein precursor, presenilin-1, or presenilin-2 gene) while familial PD genes such as Parkin, PINK-1, or DJ-1 are associated with increased oxidative stress. Also, several known genetic (e.g., apolipoprotein E\&4 variant) and environmental (e.g., metals or pesticides exposure) risk factors of sporadic $\mathrm{AD}$ and/or PD are associated with increased oxidative stress (Dei et al., 2002).

Our recent study revealed high mutation frequency in North West Indian PD population (Vinish et al., 2010) and also recently reported alterations in the lipid peroxidation and SOD profile in a separate set of PD patients, suggesting that these mutations may be related to mitochondrial dysfunction and oxidative stress. We therefore extended our study to understand if PARK2 mutations affect the antioxidant profile of such patients (Sharma et al., 2008) and thus evaluated the oxidative stress in these patients and tested whether PARK2 mutations contribute to alterations in antioxidant profile. We found that SOD levels and lipid peroxidation were higher in PD patients as compared to controls. This increase may be a compensatory response of the body to counteract the increased superoxide radicals, which are generated in these patients. We also found slight decrease in G $\mathrm{p}_{\mathrm{x}}$ activity in patients as compared to controls, which could be a result of disturbed oxidative stress (OS). Brain contains large amounts of unsaturated fatty acids, which are target for lipid peroxidation. Enhanced lipid peroxidation levels have been well reported in the postmortem brain of PD patients. MDA has also been previously reported as a potential peripheral oxidative stress marker in plasma of PD patients (Llic et al., 1999; Serra et al., 2001; Younes-Mhenni et al., 2007). Our study also revealed increase in plasma MDA levels in PD patients as compared to healthy controls, which reflects a state of OS in these patients. Therefore, the increased SOD and lipid peroxidation levels in PD patients appear to play an important role in PD pathogenesis. The elevated SOD levels have been earlier reported in blood of PD patients (Llic et al., 1999; Serra et al., 2001; Younes-Mhenni et al., 2007; Sharma et al., 2008). Besides, we also found excellent correlation between SOD and lipid peroxidation levels in the blood of these patients which can serve as a biomarker in the blood (Table 1).

PARK2, DJ-1 and PINK-1 gene mutations have been previously reported (Thomas \& Beal, 2007) in the elevation of oxidative stress. Our results partly match such antioxidant profile. Although the antioxidant profile was altered in PD patients with mutations when compared to those without mutations a larger study will determine the importance of these results. Nevertheless, this pilot study provides preliminary information which forms the basis of the investigations to follow. Based on the analysis, we conclude that alterations in SOD and lipid peroxidation levels are possibly due to increased oxidative stress in these patients or as a general compensatory response. Mutations in PARK2 might affect the oxidative machinery of PD patients but a larger study can establish this. Our study supports the involvement of oxidative stress that is implicated in the pathogenesis of PD. Perspectives for treatment of PD in the future should investigate the role of antioxidant therapy.

\section{Acknowledgements}

We acknowledge the Council of Scientific and Industrial Research (CSIR), New Delhi, for financial support to PhD scholar (Monika Vinish). We are also thankful to the valuable inputs of Mr. R.C. Goyal to statistical analysis.

\section{REFERENCES}

Buege JA, Aust SD (1978) Microsomal lipid peroxidation. Meth Ensymol 52: $306-307$.

Buhmann C, Arlt S, Kontush A, Moller-Bertram T, Sperber S (2004) Plasma and CSF markers of oxidative stress are increased in Parkinson's disease and influenced by antiparkinsonian medication. Neurobiol Dis 15: 160-170.

Dei R, Takeda A, Niwa H, Li, Nakagomi Y, Watanabe M (2002) Lipid peroxidation and advanced glycation end products in the brain in normal aging and in Alzheimer's disease. Acta Neuropathol 104: 113-122.

Dexter DT, Carter CJ, Wells FR, Javoy-Agid F, Agid Y, Less A (1989) Basal lipid peroxidation in substantia nigra is increased in Parkinson's disease. J Neurochem 52: 381-389.

Hughes AJ, Daniel SE, Kilford L, Lees AJ (1992) Accuracy of the clinical diagnosis of idiopathic Parkinson's disease, a clinical pathological study of 100 cases. J Neurol Neurosurg Psychiatry 55: 181-184.

Hung H, Lee EH (1998) MPTP produces differential oxidative stress and antioxidative responses in the nigrostriatal and mesolimbic dopaminergic pathways. Free Radic Biol Med 24: 76-84.

Hyun DH, Lee M, Halliwell B, Jenner P (2005) Effect of over expression of wild-type or mutant parkin on the cellular response induced by toxic insults. J Neurosci Res 82: 232-244.

Kitada T, Asakawa S, Hattori N, Matsumine H, Yamamura Y, Minoshima S et al. (1998) Mutations in the parkin gene cause autosomal recessive juvenile parkinsonism. Nature 392: 605-608.

Lang AE, Lozano AM (1998). Parkinson's disease. First of two parts. N Engl J Med 339: 1044-1053. 
Llic TV, Joyanoyic M, Joyicic A, Tomovic M (1999) Oxidative stress indicators are elevated in de novo Parkinson's disease patients. Parkinsonism Relat Disord 14: 141-147.

McCord JM, Fridovich I (1988) Superoxide dismutase: the first twenty years (1968-1988). Free Radic Biol Med 5: 363-369.

Vinish M, Prabhakar S, Khullar M, Verma I, Anand A (2010) Genetic screening reveals high frequency of $P A R K 2$ mutations and reduced Parkin expression conferring risk for Parkinsonism in North West India. J Neurol Neurosurg Psychiatr 81: 166-170.

Paglia DE, Valentine WN (1967) Studies on the quantitative and qualitative characterization of erythrocyte glutathione peroxidase. I Lab Clin Med 70: 158-169.

Palacino JJ, Sagi D, Goldberg MS, Krauss S, Motz C, Wacker M et al. (2004) Mitochondrial dysfunction and oxidative damage in parkindeficient mice. J Biol Chem 279: 18614-18622.

Serra JA, Demingeuz RO, Lusting ES, Guareschi EM, Famulari AL (2001) Parkinson's disease is associated with oxidative stress: com- parison of peripheral antioxidant profiles in living Parkinson's, Alzheimer's and vascular demntia patients. J Neurol Transm 108: 1135-1148.

Sharma A, Kaur P, Kumar B, Prabhakar S, Gill KD (2008) Plasma lipid peroxidation and antioxidant status of Parkinson's disease patients in the Indian population. Parkinsonism Relat Disord 14: 52-57.

Shen J, Cookson MR (2004) Mitochondria and dopamine: new insights into recessive parkinsonism. Neuron 43: 301-304.

Thomas B, Beal MF (2007) Parkinson's disease. Hum Mol Genet 16: R183-R194.

Titheradge MA (1998) The enzymatic measurement of nitrate and nitrite. In: Methods in Molecular Biology, vol. 100. Nitric oxide protocols. pp 83-90. New Jersey: Humaana Press Inc.

Younes-Mhenni S, Frih-Ayed M, Kerkeni A, Bost M, Chazot G (2007) Peripheral blood markers of oxidative stress in Parkinson's disease. Eur Neurol 58: 78-83. 